Oral

\title{
Microbiological Study of Etiological Pathogen in Early Infection of Open Fracture in Orthopedics
}

Tai Ken Meng ${ }^{1}$, Ahmad Fadzli Bin Sulong ${ }^{2}$, Nazri Bin Mohd. Yusof ${ }^{2}$

${ }^{1}$ Department of Orthopaedics, Traumatology and Rehabilitation, International Islamic University Malaysia Medical Center

${ }^{2}$ Department of Orthopaedics, Traumatology and Rehabilitation, Kulliyyah of Medicine, International Islamic University Malaysia

Presenter: Tai Ken Meng

Introduction: Infection is a dreaded complication of open fracture in the field of orthopaedics. This study was conducted to evaluate the susceptibility of microorganism involved in early infection of open fractures to current prophylactic antibiotics practice and determine the association of various characteristics to the resistance of such microorganisms. Materials and Methods: This cross sectional study was conducted from January 2015 to December 2017 in Hospital Tengku Ampuan Afzan, Kuantan, Malaysia. A total of 179 cases were collected from the operating record with 110 cases fulfilling the inclusion and exclusion criteria. The patients underwent routine debridement where culture and sensitivity were followed up. Previous notes were traced to identify the various risk factors involved and evaluated against resistance of microorganism. Statistical analysis was done with SPSS version 23 applying Pearson chi-square tests and Fisher's exact test. Results: Admission to intensive care unit, blood transfusion, antibiotic regime and duration, fracture grade, injury severity score and duration of admission were all significantly associated with resistance of microorganism ( $p<0.0001)$. This study also demonstrated $65.9 \%$ predominance of Gram-negative microorganisms. These gram-negative microorganisms were seen more in higher fracture grades, injury severity score, and longer duration of admission or antibiotics prescribed. The overall resistance to cefuroxime and gentamicin were $68.6 \%$ and $36.9 \%$ respectively. Conclusion: The shift of microorganism species to gram-negative and the high rate of resistance suggested that current antibiotic guidelines and regime needed to be fine-tuned to achieve optimal results in managing open fracture. 